\title{
Synchronization of Delayed Inertial Cohen-Grossberg Neural Networks Under Adaptive Feedback Controller
}

\author{
Qun Huang, Jinde Cao ${ }^{*}$, Qingshan Liu \\ School of Mathematics, Southeast University, Nanjing 211189, China
}

\section{ARTICLE INFO \\ Article History \\ Received 29 Feb 2020 \\ Accepted 31 Mar 2020 \\ Keywords \\ CGNNs \\ Adaptive synchronization \\ Inertial term \\ Time-varying delay}

\begin{abstract}
This paper investigates the issue on adaptive synchronization of delayed inertial Cohen-Grossberg neural networks (ICGNNs). By adopting the method of variable transformation, the addressed model, which includes the so-called inertial term, is transformed into first-order differential equations. On the basis of the well-known invariant principle of functional differential equations, a novel and analytic scheme which ensures the adaptive synchronization between the drive-response system is proposed in component form. It is worth mentioning that we only need to impose one controller to the spilt systems to realize the adaptive synchronization, which is of less conservatism. At the end of this paper, a numerical example is provided to verify the feasibility of the derived theoretical results. The established figures validate that the numerical simulations coincide well with the developed theoretical results.
\end{abstract}

(C) 2020 The Authors. Published by Atlantis Press SARL. This is an open access article distributed under the CC BY-NC 4.0 license (http://creativecommons.org/licenses/by-nc/4.0/).

\section{INTRODUCTION}

Neural networks(NNs) can be extensively applied in different fields, such as pattern recognition, parallel computing, image processing and so on. Therefore, they have been diffusely investigated in the past decades [1-7]. In order to realize these applications, it is necessary for people to study the dynamical behaviors of NNs, which greatly motivates the qualitative analysis of different dynamical behaviors [8-12]. In addition to stability, many other dynamical behaviors, including periodic oscillation, bifurcation, dissipation and synchronization, exist in real applications. Among them, the synchronization of NNs has developed into a issue of both theoretical and practical significance since synchronization is one of the most important issues related to their dynamic behaviors [13-15]. Moreover, time delays are inevitably encountered during the hardware implementation, which should be brought into the network model. Therefore, the synchronization problems of NNs with time delays have caused considerable attention and a great deal of works have been published, see [16-19] and references therein.

Particularly, the Cohen-Grossberg neural network(CGNN) model was initially established in 1983 [20]. It comprises a great deal of famous NNs, including Hopfield NNs, bidirectional associative memory NNs and cellular NNs. Consequently, the synchronization problem of CGNNs has also been proverbially studied due to their underlying applications in information processing, distributed computation and secure communication. For example, in [21-23], the synchronization problems of CGNNs with constant amplification gains and different delays were discussed. In [24], some synchronization criteria for delayed CGNNs were

\footnotetext{
*Corresponding author. Email: jdcao@seu.edu.cn
}

proposed by employing a periodic intermittent controller. In [25], Gan studied the adaptive synchronization of CGNNs with both mixed delays and unknown parameters.

Nevertheless, the existing studies mainly focus on the NNs with only first derivative of the states, and the effect caused by an inertial term is not taken into account. The introduction of the inertial term can be deemed as a key factor in generating chaotic behaviors, bifurcation and some other complicated dynamics in a networked system [26-30]. In addition, some synchronization conditions for inertial NN with or without Markovian jumping parameters were proposed in [31] and [32]. Based on the above discussion, it is obvious that the theoretical results for the synchronization of delayed ICGNNs are limited, which motivates the present research.

In the light of the well-known invariant principle, an adaptive feedback controller is provided to achieve the synchronization goal. Compared with previous research, the main contributions of the work are summarized in three perspectives: (i) The introduction of inertial term makes this problem more challenging, which has been successfully resolved based on the variable transformation method. (ii) We only need to impose one controller to the spilt systems, which is of less conservatism. (iii) The model considered in this paper is rather general, thus it comprises many existing results as its special cases.

The remaining part of the paper is organized as follows. The model description, necessary definition, lemma as well as assumptions are given in Section 2. A novel and analytic adaptive controller is presented in Section 3 to realize the synchronization of delayed ICGNNs. Moreover, an illustrative example is presented to validate the feasibility of the developed synchronization strategy in Section 4. At last, the conclusion of this work is made in Section 5. 


\section{MODEL DESCRIPTION AND PRELIMINARIES}

In this paper, the delayed ICGNNs model can be described by the following differential equations:

$$
\begin{aligned}
\frac{d^{2} u_{i}(t)}{d t^{2}}= & -\beta_{i} \frac{d u_{i}(t)}{d t}-\alpha_{i}\left(u_{i}(t)\right)\left[h_{i}\left(u_{i}(t)\right)-\sum_{j=1}^{n} a_{i j} f_{j}\left(u_{j}(t)\right)\right. \\
& \left.-\sum_{j=1}^{n} b_{i j} f_{j}\left(u_{j}(t-\tau(t))\right)+J_{i}\right]
\end{aligned}
$$

for $i \in\{1,2, \cdots, n\}$. In which $u_{i}(t)$ denotes the state of the $i$ th neuron at time $t$ and the second derivative of $u_{i}(t)$ represents the inertial term, $a_{i j}$ and $b_{i j}$ stand for the connection strength and the timevarying delay connection strength, respectively. $f_{j}(\cdot)$ represents the the activation function, $\beta_{i}>0$ is a constant, $\alpha_{i}(\cdot)$ is an amplification function, $h_{i}(\cdot)$ denotes an appropriate behaved function, $J_{i}$ stands for the external input and $\tau(t)$ represents the time-varying delay.

Considering (1) as the drive system, the corresponding response system is devised as

$$
\begin{aligned}
\frac{d^{2} x_{i}(t)}{d t^{2}}= & -\beta_{i} \frac{d x_{i}(t)}{d t}-\alpha_{i}\left(x_{i}(t)\right)\left[h_{i}\left(x_{i}(t)\right)-\sum_{j=1}^{n} a_{i j} f_{j}\left(x_{j}(t)\right)\right. \\
& \left.-\sum_{j=1}^{n} b_{i j} f_{j}\left(x_{j}(t-\tau(t))\right)+J_{i}\right]+U_{i}(t)
\end{aligned}
$$

where $U_{i}(t)$ is an appropriate controller that will be designed in the sequel.

The variable transformation is introduced as

$$
\begin{aligned}
& v_{i}(t)=\frac{d u_{i}(t)}{d t}+\delta_{i} u_{i}(t), i=1,2, \cdots, n, \\
& y_{i}(t)=\frac{d x_{i}(t)}{d t}+\delta_{i} x_{i}(t), i=1,2, \cdots, n,
\end{aligned}
$$

where $\delta_{i}>0$ is a constant.

Then, the above two systems are equivalent to

$$
\left\{\begin{aligned}
\frac{d u_{i}(t)}{d t}= & -\delta_{i} u_{i}(t)+v_{i}(t) \\
\frac{d v_{i}(t)}{d t}= & -\delta_{i}\left(\delta_{i}-\beta_{i}\right) u_{i}(t)+\left(\delta_{i}-\beta_{i}\right) v_{i}(t)-\alpha_{i}\left(u_{i}(t)\right) \\
& \times\left[h_{i}\left(u_{i}(t)\right)-\sum_{j=1}^{n} a_{i j} f_{j}\left(u_{j}(t)\right)-\sum_{j=1}^{n} b_{i j}\right. \\
& \left.\times f_{j}\left(u_{j}(t-\tau(t))\right)+J_{i}\right]
\end{aligned}\right.
$$

and

$$
\left\{\begin{aligned}
\frac{d x_{i}(t)}{d t}= & -\delta_{i} x_{i}(t)+y_{i}(t) \\
\frac{d y_{i}(t)}{d t}= & -\delta_{i}\left(\delta_{i}-\beta_{i}\right) x_{i}(t)+\left(\delta_{i}-\beta_{i}\right) y_{i}(t)-\alpha_{i}\left(x_{i}(t)\right) \\
& \times\left[h_{i}\left(x_{i}(t)\right)-\sum_{j=1}^{n} a_{i j} f_{j}\left(x_{j}(t)\right)-\sum_{j=1}^{n} b_{i j}\right. \\
& \left.\times f_{j}\left(x_{j}(t-\tau(t))\right)+J_{i}\right]+U_{i}(t) .
\end{aligned}\right.
$$

Denote the error signals as $e_{1 i}(t)=x_{i}(t)-u_{i}(t)$ and $e_{2 i}(t)=y_{i}(t)-$ $v_{i}(t)$. Then, the error dynamics between the uncontrolled system (3) and the controlled system (4) are derived as follows:

$$
\left\{\begin{aligned}
\frac{d e_{1 i}(t)}{d t}= & -\delta_{i} e_{1 i}(t)+e_{2 i}(t), \\
\frac{d e_{2 i}(t)}{d t}= & -\delta_{i}\left(\delta_{i}-\beta_{i}\right) e_{1 i}(t)+\left(\delta_{i}-\beta_{i}\right) e_{2 i}(t)-\left(\alpha_{i}\left(x_{i}(t)\right)\right. \\
& \left.\times h_{i}\left(x_{i}(t)\right)-\alpha_{i}\left(u_{i}(t)\right) h_{i}\left(u_{i}(t)\right)\right)+\alpha_{i}\left(x_{i}(t)\right) \\
& \times \sum_{j=1}^{n} a_{i j}\left(f_{j}\left(x_{j}(t)\right)-f_{j}\left(u_{j}(t)\right)\right)+\alpha_{i}\left(x_{i}(t)\right) \\
& \times \sum_{j=1}^{n} b_{i j}\left(f_{j}\left(x_{j}(t-\tau(t))\right)-f_{j}\left(u_{j}(t-\tau(t))\right)\right) \\
& +\left(\alpha_{i}\left(x_{i}(t)\right)-\alpha_{i}\left(u_{i}(t)\right)\right) \sum_{j=1}^{n} a_{i j} f_{j}\left(u_{j}(t)\right) \\
& +\left(\alpha_{i}\left(x_{i}(t)\right)-\alpha_{i}\left(u_{i}(t)\right)\right) \sum_{j=1}^{n} b_{i j} f_{j}\left(u_{j}(t-\tau(t))\right) \\
& -\left(\alpha_{i}\left(x_{i}(t)\right)-\alpha_{i}\left(u_{i}(t)\right)\right) J_{i}+U_{i}(t) .
\end{aligned}\right.
$$

To proceed further, the following hypotheses are utilized: $\left(A_{1}\right)$ : There exist positive constants $\bar{\alpha}_{i}$ and $M_{i}$ such that

$$
0 \leq \alpha_{i}\left(u_{i}\right) \leq \bar{\alpha}_{i}, \quad\left|\alpha_{i}\left(u_{i}\right)-\alpha_{i}\left(v_{i}\right)\right| \leq M_{i}\left|u_{i}-v_{i}\right|,
$$

for all $u_{i}, v_{i} \in \mathbb{R}, i \in\{1,2, \cdots, n\}$.

$\left(A_{2}\right)$ : There exist positive constants $l_{i}$ and $f_{i}$ such that the activation functions $f_{i}(\cdot)$ satisfy

$$
\left|f_{i}\left(u_{i}\right)\right| \leq f_{i}, \quad\left|f_{i}\left(u_{i}\right)-f_{i}\left(v_{i}\right)\right| \leq l_{i}\left|u_{i}-v_{i}\right|
$$

where $u_{i}, v_{i} \in \mathbb{R}, i \in\{1,2, \cdots, n\}$.

$\left(A_{3}\right)$ : There exist positive constants $\gamma_{i}$ such that

$$
\left|\alpha_{i}\left(u_{i}\right) h_{i}\left(u_{i}\right)-\alpha_{i}\left(v_{i}\right) h_{i}\left(v_{i}\right)\right| \leq \gamma_{i}\left|u_{i}-v_{i}\right|
$$

where $u_{i}, v_{i} \in \mathbb{R}, i \in\{1,2, \cdots, n\}$.

$\left(A_{4}\right)$ : The time-varying transmission delay $\tau(t)$ is supposed to satisfy

$$
0<\tau(t) \leq \tau, \quad \dot{\tau}(t) \leq p<1 .
$$

Before moving on, we present the following definition and lemma:

Definition 1. The drive system (1) and the response system (2) is said to achieve synchronization, if the trivial solution of the error system (5) is asymptotically stable, i.e.,

$$
\lim _{t \rightarrow \infty} e_{1 i}(t)=\lim _{t \rightarrow \infty}\left(x_{i}(t)-u_{i}(t)\right)=0 . \quad i=1,2, \cdots, n .
$$

Remark 1. Since the drive-response systems (1) and (2) are equivalent to (3) and (4), respectively, the synchronization problem between (1) and (2) can be regarded as the synchronization problem between (3) and (4). In addition, according to the variable transformation, one can see that $e_{2 i}(t)$ just plays the role of adjoint variable. Thus we only need to consider the dynamics of $e_{1 i}(t)$ when investigating the synchronization problem between systems (1) and (2).

Lemma 2. [25] For any vectors $u, v \in \mathbb{R}^{n}$, and any positive definite matrix $\Xi \in \mathbb{R}^{n \times n}$, the following matrix inequality always holds:

$$
2 u^{T} v \leq u^{T} \Xi u+v^{T} \Xi^{-1} v .
$$




\section{MAIN RESULTS}

In this part, a new criterion is presented to achieve the adaptive synchronization of ICGNNs with time-varying delay. Besides, the corresponding corollaries of inertial NNs and delayed CGNNs are proposed.

Theorem 1. If the hypotheses $\left(A_{1}\right)-\left(A_{4}\right)$ hold, then the driveresponse delayed ICGNNs (1) and (2) are synchronous with the adaptive feedback controller as

$$
U_{i}(t)=\xi_{i} e_{2 i}(t)+\eta_{i}\left|e_{1 i}(t)\right| \operatorname{sign}\left(e_{2 i}(t)\right)
$$

the feedback strengths $\xi_{i}$ and $\eta_{i}(i=1,2, \cdots, n)$ are adapted in the light of the following update laws, respectively:

$$
\dot{\xi}_{i}=-\varepsilon_{i} e_{2 i}^{2}(t), \quad \dot{\eta}_{i}=-\theta_{i}\left|e_{1 i}(t)\right|\left|e_{2 i}(t)\right|,
$$

where $\varepsilon_{i}$ and $\theta_{i}(i=1,2, \cdots, n)$ are any positive scalars.

Proof. Constructing the following Lyapunov functional:

$$
\begin{aligned}
V(t)= & \frac{1}{2} \sum_{i=1}^{n}\left[e_{1 i}^{2}(t)+e_{2 i}^{2}(t)+\frac{1}{1-p} \sum_{j=1}^{n} \bar{\alpha}_{i} l_{j}\left|b_{i j}\right|\right. \\
& \left.\times \int_{t-\tau(t)}^{t} e_{1 j}^{2}(s) d s+\frac{1}{\varepsilon_{i}}\left(\xi_{i}+\lambda_{1 i}\right)^{2}+\frac{1}{\theta_{i}}\left(\eta_{i}+\lambda_{2 i}\right)^{2}\right],
\end{aligned}
$$

where $\lambda_{1 i}$ and $\lambda_{2 i}$ are scalars to be confirmed.

Calculating $\dot{V}(t)$ along the trajectory of error system (5) results in

$$
\begin{aligned}
\dot{V}(t)= & \sum_{i=1}^{n}\left[e_{1 i}(t) \dot{e}_{1 i}(t)+e_{2 i}(t) \dot{e}_{2 i}(t)+\frac{1}{2(1-p)} \sum_{j=1}^{n} \bar{\alpha}_{i} l_{j}\right. \\
& \times\left|b_{i j}\right| e_{1 j}^{2}(t)-\frac{1-\dot{\tau}(t)}{2(1-p)} \sum_{j=1}^{n} \bar{\alpha}_{i} l_{j}\left|b_{i j}\right| e_{1 j}^{2}(t-\tau(t)) \\
& \left.+\frac{1}{\varepsilon_{i}}\left(\xi_{i}+\lambda_{1 i}\right) \dot{\xi}_{i}+\frac{1}{\theta_{i}}\left(\eta_{i}+\lambda_{2 i}\right) \dot{\eta}_{i}\right] \\
= & \sum_{i=1}^{n}\left[e_{1 i}(t)\left(-\delta_{i} e_{1 i}(t)+e_{2 i}(t)\right)\right. \\
& +e_{2 i}(t)\left(-\delta_{i}\left(\delta_{i}-\beta_{i}\right) e_{1 i}(t)+\left(\delta_{i}-\beta_{i}\right) e_{2 i}(t)\right. \\
& -\left(\alpha_{i}\left(x_{i}(t)\right) h_{i}\left(x_{i}(t)\right)-\alpha_{i}\left(u_{i}(t)\right) h_{i}\left(u_{i}(t)\right)\right) \\
& +\alpha_{i}\left(x_{i}(t)\right) \sum_{j=1}^{n} a_{i j}\left(f_{j}\left(x_{j}(t)\right)-f_{j}\left(u_{j}(t)\right)\right)+\alpha_{i}\left(x_{i}(t)\right) \\
& \left.+\left(\alpha_{i}\left(x_{i}(t)\right)-\alpha_{i}\left(u_{i}(t)\right)\right) J_{i}+U_{i}(t)\right) \\
& +\left(\sum_{j=1}^{n} b_{i j}\left(f_{j}\left(x_{j}(t-\tau(t))\right)-f_{j}\left(u_{j}(t-\tau(t))\right)\right)\right. \\
& +\left(\alpha_{i}\left(x_{i}(t)\right)-\alpha_{i}\left(u_{i}(t)\right)\right) \sum_{j=1}^{n} a_{i j} f_{j}\left(u_{j}(t)\right) \\
n & \left.\alpha_{i}\left(u_{i}(t)\right)\right) \sum_{i j} f_{j}\left(u_{j}(t-\tau(t))\right) \\
&
\end{aligned}
$$

$$
\begin{aligned}
& +\frac{1}{2(1-p)} \sum_{j=1}^{n} \bar{\alpha}_{i} l_{j}\left|b_{i j}\right| e_{1 j}^{2}(t)-\frac{1-\dot{\tau}(t)}{2(1-p)} \sum_{j=1}^{n} \bar{\alpha}_{i} l_{j} \\
& \left.\times\left|b_{i j}\right| e_{1 j}^{2}(t-\tau(t))-\left(\xi_{i}+\lambda_{1 i}\right) e_{2 i}^{2}(t)-\left(\eta_{i}+\lambda_{2 i}\right)\left|e_{1 i}(t)\right|\left|e_{2 i}(t)\right|\right] .
\end{aligned}
$$

Considering the assumptions $\left(A_{1}\right)-\left(A_{4}\right)$ and the adaptive feedback controller (6), $\dot{V}(t)$ undergoes the following estimation:

$$
\begin{aligned}
\dot{V}(t) \leq & \sum_{i=1}^{n}\left[-\delta_{i} e_{1 i}^{2}(t)+e_{1 i}(t) e_{2 i}(t)-\delta_{i}\left(\delta_{i}-\beta_{i}\right) e_{1 i}(t) e_{2 i}(t)\right. \\
& +\left(\delta_{i}-\beta_{i}\right) e_{2 i}^{2}(t)+\gamma_{i}\left|e_{1 i}(t)\right|\left|e_{2 i}(t)\right|+\left|e_{2 i}(t)\right| \bar{\alpha}_{i} \\
& \times \sum_{j=1}^{n}\left|a_{i j}\right| l_{j}\left|e_{1 j}(t)\right|+\left|e_{2 i}(t)\right| \bar{\alpha}_{i} \sum_{j=1}^{n}\left|b_{i j}\right| l_{j}\left|e_{1 j}(t-\tau(t))\right| \\
& +M_{i}\left|e_{2 i}(t)\right|\left|e_{1 i}(t)\right| \sum_{j=1}^{n}\left|a_{i j}\right| f_{j}+M_{i}\left|e_{2 i}(t)\right|\left|e_{1 i}(t)\right| \\
& \times \sum_{j=1}^{n}\left|b_{i j}\right| f_{j}+M_{i}\left|e_{2 i}(t)\right|\left|e_{1 i}(t)\right|\left|J_{i}\right|+\frac{1}{2(1-p)} \\
& \times \sum_{j=1}^{n} \bar{\alpha}_{i} l_{j}\left|b_{i j}\right| e_{1 j}^{2}(t)-\frac{1}{2} \sum_{j=1}^{n} \bar{\alpha}_{i} l_{j}\left|b_{i j}\right| e_{1 j}^{2}(t-\tau(t)) \\
& \left.-\lambda_{1 i} e_{2 i}^{2}(t)-\lambda_{2 i}\left|e_{1 i}(t)\right|\left|e_{2 i}(t)\right|\right] .
\end{aligned}
$$

According to Lemma 2, one can derive thatt

$$
\begin{aligned}
\sum_{i=1}^{n}\left|e_{2 i}(t)\right| \bar{\alpha}_{i} & \sum_{j=1}^{n}\left|a_{i j}\right| l_{j}\left|e_{1 j}(t)\right| \\
& \leq \frac{1}{2} \sum_{i=1}^{n} \sum_{j=1}^{n} \bar{\alpha}_{i}\left|a_{i j}\right| l_{j}\left(e_{2 i}^{2}(t)+e_{1 j}^{2}(t)\right), \\
\sum_{i=1}^{n}\left|e_{2 i}(t)\right| \bar{\alpha}_{i} \quad & \sum_{j=1}^{n}\left|a_{i j}\right| l_{j}\left|e_{1 j}(t-\tau(t))\right| \\
& \leq \frac{1}{2} \sum_{i=1}^{n} \sum_{j=1}^{n} \bar{\alpha}_{i}\left|a_{i j}\right| l_{j}\left(e_{2 i}^{2}(t)+e_{1 j}^{2}(t-\tau(t))\right) .
\end{aligned}
$$

Substituting (10) into the right side of (9), one can further obtain that

$$
\begin{aligned}
\dot{V}(t) \leq & \sum_{i=1}^{n}\left[-\delta_{i} e_{1 i}^{2}(t)+\left(\delta_{i}-\beta_{i}\right) e_{2 i}^{2}(t)+\left|1-\delta_{i}^{2}+\delta_{i} \beta_{i}\right|\right. \\
& \times\left|e_{1 i}(t)\right|\left|e_{2 i}(t)\right|+\gamma_{i}\left|e_{1 i}(t)\right|\left|e_{2 i}(t)\right|+\frac{1}{2} \bar{\alpha}_{i} \\
& \times \sum_{j=1}^{n}\left(\left|a_{i j}\right|+\left|b_{i j}\right|\right) l_{j} e_{2 i}^{2}(t)+\frac{1}{2} \sum_{j=1}^{n} \bar{\alpha}_{j}\left|a_{j i}\right| l_{i} e_{1 i}^{2}(t) \\
& +M_{i}\left(\sum_{j=1}^{n}\left(\left|a_{i j}\right|+\left|b_{i j}\right|\right) f_{j}+\left|J_{i}\right|\right)\left|e_{1 i}(t)\right|\left|e_{2 i}(t)\right| \\
& \left.+\frac{1}{2(1-p)} \sum_{j=1}^{n} \bar{\alpha}_{j}\left|b_{j i}\right| l_{i} e_{1 i}^{2}(t)-\lambda_{1 i} e_{2 i}^{2}(t)-\lambda_{2 i} \times\left|e_{1 i}(t)\right|\left|e_{2 i}(t)\right|\right] \\
= & \sum_{i=1}^{n}\left[\left(\frac{1}{2} \sum_{j=1}^{n} \bar{\alpha}_{j}\left|a_{j i}\right| l_{i}+\frac{1}{2(1-p)} \sum_{j=1}^{n} \bar{\alpha}_{j}\left|b_{j i}\right| l_{i}-\delta_{i}\right)\right. \\
& \times e_{1 i}^{2}(t)+\left(\frac{1}{2} \bar{\alpha}_{i} \sum_{j=1}^{n}\left(\left|a_{i j}\right|+\left|b_{i j}\right|\right) l_{j}+\delta_{i}-\beta_{i}-\lambda_{1 i}\right)
\end{aligned}
$$




$$
\begin{aligned}
& \times e_{2 i}^{2}(t)+\left(\left|1-\delta_{i}^{2}+\delta_{i} \beta_{i}\right|+\gamma_{i}+M_{i}\left(\sum_{j=1}^{n}\left(\left|a_{i j}\right|+\left|b_{i j}\right|\right)\right.\right. \\
& \left.\left.\left.f_{j}+\left|J_{i}\right|\right)-\lambda_{2 i}\right)\left|e_{1 i}(t)\right|\left|e_{2 i}(t)\right|\right] .
\end{aligned}
$$

Letting

$$
\begin{aligned}
& \delta_{i}=\frac{1}{2} \sum_{j=1}^{n} \bar{\alpha}_{j}\left|a_{j i}\right| l_{i}+\frac{1}{2(1-p)} \sum_{j=1}^{n} \bar{\alpha}_{j}\left|b_{j i}\right| l_{i}+1, \\
& \lambda_{1 i}=\frac{1}{2} \bar{\alpha}_{i} \sum_{j=1}^{n}\left(\left|a_{i j}\right|+\left|b_{i j}\right|\right) l_{j}+\delta_{i}-\beta_{i}, \\
& \lambda_{2 i}=\left|1-\delta_{i}^{2}+\delta_{i} \beta_{i}\right|+\gamma_{i}+M_{i}\left(\sum_{j=1}^{n}\left(\left|a_{i j}\right|+\left|b_{i j}\right|\right) f_{j}+\left|J_{i}\right|\right) .
\end{aligned}
$$

In light of (11) and (12), one has

$$
\dot{V}(t) \leq \sum_{i=1}^{n}\left(-e_{1 i}^{2}(t)\right)=-e_{1}^{T}(t) e_{1}(t)
$$

where $e_{1}(t)=\left(e_{11}(t), e_{12}(t), \cdots, e_{1 n}(t)\right)^{T}$. It is apparent that $\dot{V}(t)=$ 0 if and only if $e_{1}(t)=0$. Based on Definition 1 and the famous invariant principle, no matter what the initial value is, the trajectory of $e_{1}(t)$ would converge asymptotically to the largest invariant set $E=\left\{e_{1}(t)=0\right\}$ contained in $\lim \dot{V}(t)=0$, which implies the adaptive synchronization can be reached.

Remark 2. The synchronization issue of delayed ICGNNs are successfully resolved in Theorem 1 . By means of variable transformation method, the original inertial system is split into two subsystems. By imposing an adaptive controller to subsystem 2, Theorem 1 has presented a componentwise scheme to assure the adaptive synchronization.

If we take $\alpha_{i}\left(u_{i}(t)\right)=1$ and $h_{i}\left(u_{i}(t)\right)=c_{i} u_{i}(t)$, then the model (1) degenerates to

$$
\begin{aligned}
\frac{d^{2} u_{i}(t)}{d t^{2}}= & -\beta_{i} \frac{d u_{i}(t)}{d t}-c_{i} u_{i}(t)+\sum_{j=1}^{n} a_{i j} f_{j}\left(u_{j}(t)\right)+\sum_{j=1}^{n} b_{i j} \\
& \times f_{j}\left(u_{j}(t-\tau(t))\right)-J_{i}, \quad i=1,2, \cdots, n .
\end{aligned}
$$

As a consequence, the corresponding response system is

$$
\begin{aligned}
\frac{d^{2} x_{i}(t)}{d t^{2}}= & -\beta_{i} \frac{d x_{i}(t)}{d t}-c_{i} x_{i}(t)+\sum_{j=1}^{n} a_{i j} f_{j}\left(x_{j}(t)\right)+\sum_{j=1}^{n} b_{i j} \\
& \times f_{j}\left(x_{j}(t-\tau(t))\right)-J_{i}+U_{i}(t) .
\end{aligned}
$$

Following the aforementioned variable transformation, the above two systems can be rewritten as

$$
\left\{\begin{aligned}
\frac{d u_{i}(t)}{d t}= & -\delta_{i} u_{i}(t)+v_{i}(t), \\
\frac{d v_{i}(t)}{d t}= & -\delta_{i}\left(\delta_{i}-\beta_{i}\right) u_{i}(t)+\left(\delta_{i}-\beta_{i}\right) v_{i}(t)-c_{i} u_{i}(t) \\
& +\sum_{j=1}^{n} a_{i j} f_{j}\left(u_{j}(t)\right)+\sum_{j=1}^{n} b_{i j} f_{j}\left(u_{j}(t-\tau(t))\right)-J_{i},
\end{aligned}\right.
$$

and

$$
\left\{\begin{aligned}
\frac{d x_{i}(t)}{d t}= & -\delta_{i} x_{i}(t)+y_{i}(t), \\
\frac{d y_{i}(t)}{d t}= & -\delta_{i}\left(\delta_{i}-\beta_{i}\right) x_{i}(t)+\left(\delta_{i}-\beta_{i}\right) y_{i}(t)-c_{i} x_{i}(t) \\
& +\sum_{j=1}^{n} a_{i j} f_{j}\left(x_{j}(t)\right)+\sum_{j=1}^{n} b_{i j} f_{j}\left(x_{j}(t-\tau(t))\right)-J_{i}+U_{i}(t) .
\end{aligned}\right.
$$

Following the same line as in Theorem 1, the following corollary can be readily obtained:

Corollary 1. Suppose that the assumptions $\left(A_{2}\right)$ and $\left(A_{4}\right)$ hold, the drive-response delayed inertial NNs (14) and (15) are synchronous with the adaptive controller as

$$
U_{i}(t)=\xi_{i} e_{2 i}(t)+\eta_{i}\left|e_{1 i}(t)\right| \operatorname{sign}\left(e_{2 i}(t)\right),
$$

the feedback strengths $\xi_{i}$ and $\eta_{i}(i=1,2, \cdots, n)$ are adapted in the light of the following update laws, respectively:

$$
\dot{\xi}_{i}=-\varepsilon_{i} e_{2 i}^{2}(t), \quad \dot{\eta}_{i}=-\theta_{i}\left|e_{1 i}(t)\right|\left|e_{2 i}(t)\right|,
$$

where $\varepsilon_{i}$ and $\theta_{i}(i=1,2, \cdots, n)$ are arbitrary positive scalars.

Letting

$$
\begin{aligned}
& \delta_{i}=\frac{1}{2} \sum_{j=1}^{n}\left|a_{j i}\right| l_{i}+\frac{1}{2(1-p)} \sum_{j=1}^{n}\left|b_{j i}\right| l_{i}+1, \\
& \lambda_{1 i}=\frac{1}{2} \sum_{j=1}^{n}\left(\left|a_{i j}\right|+\left|b_{i j}\right|\right) l_{j}+\delta_{i}-\beta_{i}, \\
& \lambda_{2 i}=\left|1-\delta_{i}^{2}+\delta_{i} \beta_{i}-c_{i}\right| .
\end{aligned}
$$

Remark 3. In [31], the authors discussed the synchronization problem of delayed inertial NNs based on the feedback controller. Compared with the model in that paper, Corollary 1 does not impose two controllers to the split drive system, which is of less conservatism.

When $\frac{d^{2} u_{i}(t)}{d t^{2}}=0, \beta_{i}=1$, the system (1) is further degenerated to

$$
\begin{aligned}
\frac{d u_{i}(t)}{d t}=- & \alpha_{i}\left(u_{i}(t)\right)\left[h_{i}\left(u_{i}(t)\right)-\sum_{j=1}^{n} a_{i j} f_{j}\left(u_{j}(t)\right)-\sum_{j=1}^{n} b_{i j}\right. \\
& \left.\times f_{j}\left(u_{j}(t-\tau(t))\right)+J_{i}\right], \quad i=1,2, \cdots, n .
\end{aligned}
$$

For the drive system (20), the corresponding response system can be designed as

$$
\begin{aligned}
\frac{d x_{i}(t)}{d t}= & -\alpha_{i}\left(x_{i}(t)\right)\left[h_{i}\left(x_{i}(t)\right)-\sum_{j=1}^{n} a_{i j} f_{j}\left(x_{j}(t)\right)-\sum_{j=1}^{n} b_{i j}\right. \\
& \left.\times f_{j}\left(x_{j}(t-\tau(t))\right)+J_{i} \times\right]+U_{i}(t) .
\end{aligned}
$$

Analogously, we denote the error signal as $e_{i}(t)=x_{i}(t)-u_{i}(t)$. By constructing a slightly different Lypunov functional $V(t)=\frac{1}{2}$ $\sum_{i=1}^{n}\left[e_{i}^{2}(t)+\frac{1}{1-p} \sum_{j=1}^{n} \bar{\alpha}_{i} l_{j}\left|b_{i j}\right| \int_{t-\tau(t)}^{t} e_{j}^{2}(s) d s+\frac{1}{\mu_{i}}\left(\delta_{i}+\lambda_{i}\right)^{2}\right]$ and 
following the similar way as in Theorem 1, one can easily derive the adaptive synchronization criterion for systems (20) and (21), which is described in the following corollary without detailed proof.

Corollary 2. If the hypotheses $\left(A_{1}\right)-\left(A_{4}\right)$ are satisfied, then the drive-response delayed CGNNs (20) and (21) are synchronous with the controller devised as

$$
U_{i}(t)=\delta_{i} e_{i}(t)
$$

the parameter $\delta_{i}(i=1,2, \cdots, n)$ is adapted in light of the following update law:

$$
\dot{\delta}_{i}=-\mu_{i} e_{i}^{2}(t)
$$

with $\mu_{i}(i=1,2, \cdots, n)$ are any positive scalars.

Letting

$$
\begin{aligned}
\lambda_{i}= & \frac{1}{2} \sum_{j=1}^{n}\left(\bar{\alpha}_{i}\left|a_{i j}\right| l_{j}+\bar{\alpha}_{i}\left|b_{i j}\right| l_{j}+\bar{\alpha}_{j}\left|a_{j i}\right| l_{i}\right)+M_{i} \\
& \times\left(\sum_{j=1}^{n}\left(\left|a_{i j}+b_{i j}\right|\right) f_{j}+\left|J_{i}\right|\right)+\frac{1}{2(1-p)} \sum_{j=1}^{n} \bar{\alpha}_{j}\left|b_{j i}\right| l_{i}+\gamma_{i}+1 .
\end{aligned}
$$

Remark 4. In [16] and [21], the authors studied the synchronization of CGNNs with constant amplification gains, which could be deemed as a special case of Corollary 2. From the above discussion, it is obvious that the model considered in this paper is quite general and our results effectually improve several known ones.

\section{A NUMERICAL EXAMPLE}

Consider the following two-dimensional ICGNNs with timevarying delay:

$$
\begin{aligned}
\frac{d^{2} u_{i}(t)}{d t^{2}}= & -\beta_{i} \frac{d u_{i}(t)}{d t}-\alpha_{i}\left(u_{i}(t)\right)\left[h_{i}\left(u_{i}(t)\right)-\sum_{j=1}^{2} a_{i j}\right. \\
& \left.\times f_{j}\left(u_{j}(t)\right)-\sum_{j=1}^{2} b_{i j} f_{j}\left(u_{j}(t-\tau(t))\right)+J_{i}\right],
\end{aligned}
$$

where $i=1,2$. The parameters of system (23) are set as $\beta_{1}=\beta_{2}=$ $1, a_{11}=-0.6, a_{12}=0.3, a_{21}=0.4, a_{22}=-0.1, b_{11}=0.1, b_{12}=$ $-0.2, b_{21}=0.2, b_{22}=-0.05 . \tau(t)=0.5+0.5 \sin t, J_{1}=J_{2}=0.2$. In addition, $\alpha_{1}\left(u_{1}\right)=2-\frac{1}{1+u_{1}^{2}}, \alpha_{2}\left(u_{2}\right)=2+\frac{1}{1+u_{2}^{2}}, h_{i}\left(u_{i}\right)=u_{i}$, $f_{i}\left(u_{i}\right)=\tanh \left(u_{i}\right)$ for $i=1,2$.

The corresponding response system is presented as

$$
\begin{aligned}
\frac{d^{2} x_{i}(t)}{d t^{2}}= & -\beta_{i} \frac{d x_{i}(t)}{d t}-\alpha_{i}\left(x_{i}(t)\right)\left[h_{i}\left(x_{i}(t)\right)-\sum_{j=1}^{2} a_{i j}\right. \\
& \left.\times f_{j}\left(x_{j}(t)\right)-\sum_{j=1}^{2} b_{i j} f_{j}\left(x_{j}(t-\tau(t))\right)+J_{i}\right]+U_{i}(t) .
\end{aligned}
$$

By adopting the variable transformation, the above two systems can be rewritten as

$$
\left\{\begin{aligned}
\frac{d u_{i}(t)}{d t}= & -\delta_{i} u_{i}(t)+v_{i}(t) \\
\frac{d v_{i}(t)}{d t}= & -\delta_{i}\left(\delta_{i}-\beta_{i}\right) u_{i}(t)+\left(\delta_{i}-\beta_{i}\right) v_{i}(t)-\alpha_{i}\left(u_{i}(t)\right) \\
& \times\left[h_{i}\left(u_{i}(t)\right)-\sum_{j=1}^{2} a_{i j} f_{j}\left(u_{j}(t)\right)-\sum_{j=1}^{2} b_{i j}\right. \\
& \left.\times f_{j}\left(u_{j}(t-\tau(t))\right)+J_{i}\right]
\end{aligned}\right.
$$

and

$$
\left\{\begin{aligned}
\frac{d x_{i}(t)}{d t}= & -\delta_{i} x_{i}(t)+y_{i}(t) \\
\frac{d y_{i}(t)}{d t}= & -\delta_{i}\left(\delta_{i}-\beta_{i}\right) x_{i}(t)+\left(\delta_{i}-\beta_{i}\right) y_{i}(t)-\alpha_{i}\left(x_{i}(t)\right) \\
& \times\left[h_{i}\left(x_{i}(t)\right)-\sum_{j=1}^{2} a_{i j} f_{j}\left(x_{j}(t)\right)-\sum_{j=1}^{2} b_{i j}\right. \\
& \left.\times f_{j}\left(x_{j}(t-\tau(t))\right)+J_{i}\right]+U_{i}(t) .
\end{aligned}\right.
$$

It is obvious that $1 \leq \alpha_{1}\left(u_{1}\right) \leq 2,2 \leq \alpha_{2}\left(u_{2}\right) \leq 3$, which implies $\bar{\alpha}_{1}=2, \bar{\alpha}_{2}=3$. For any $u, v \in \mathbb{R}$, one has

$$
\begin{aligned}
\left|\alpha_{i}(u)-\alpha_{i}(v)\right| & =\left|\frac{1}{1+u^{2}}-\frac{1}{1+v^{2}}\right| \\
& \leq \frac{|u|+|v|}{\left(1+u^{2}\right)\left(1+v^{2}\right)}|u-v| \leq|u-v|,
\end{aligned}
$$

for $i=1,2$. Hence we take $M_{1}=M_{2}=1$.

Moreover, for any $u, v \in \mathbb{R}$, we also have

$$
\begin{aligned}
\left|\alpha_{i}(u) h_{i}(u)-\alpha_{i}(v) h_{i}(v)\right| & \leq 2|u-v|+\left|\frac{u}{1+u^{2}}-\frac{v}{1+v^{2}}\right| \\
& \leq 3|u-v|,
\end{aligned}
$$

for $i=1,2$. Thus we choose $\gamma_{1}=\gamma_{2}=3$.

Since $f_{i}\left(u_{i}\right)=\tanh \left(u_{i}\right), \tau(t)=0.5+0.5 \sin t$, one can set $f_{i}=l_{i}=$ $1(i=1,2)$ and $p=0.5$.

Then the assumptions $\left(A_{1}\right)-\left(A_{4}\right)$ are all satisfied. According to (12), after a simple calculation, we take $\delta_{1}=3, \delta_{2}=2, \lambda_{11}=3.2$, $\lambda_{12}=2.125, \lambda_{21}=9.4, \lambda_{22}=4.95$. According to Theorem 1 , the drive-response systems (24) and (25) can achieve synchronization. Figure 1 depicts the state trajectories of systems (24) and (25). Figure 2 further depicts the synchronization errors $e_{1 i}(t)(i=1,2)$ between the uncontrolled system and the controlled system. The dynamic behaviors of control parameters $\xi_{i}$ and $\eta_{i}(i=1,2)$ are illustrated in Figure 3. It is obvious that the numerical simulations coincide well with the developed theoretical results.

\section{CONCLUSTION}

Generally, the synchronization problem of delayed ICGNNs has been addressed in this work. The introduction of inertial term 

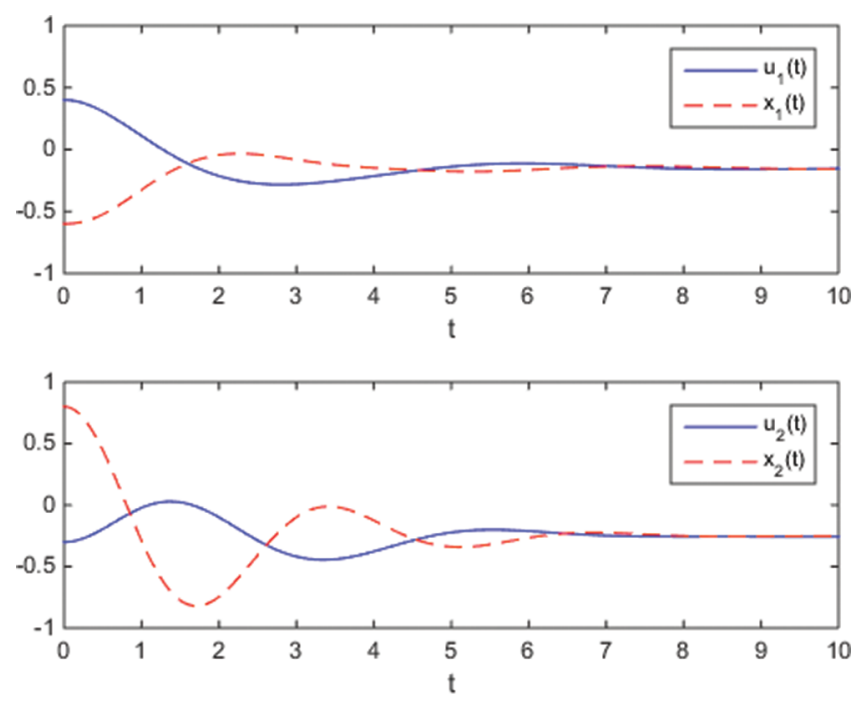

Figure 1 State trajectories of drive-response systems (24) and (25).

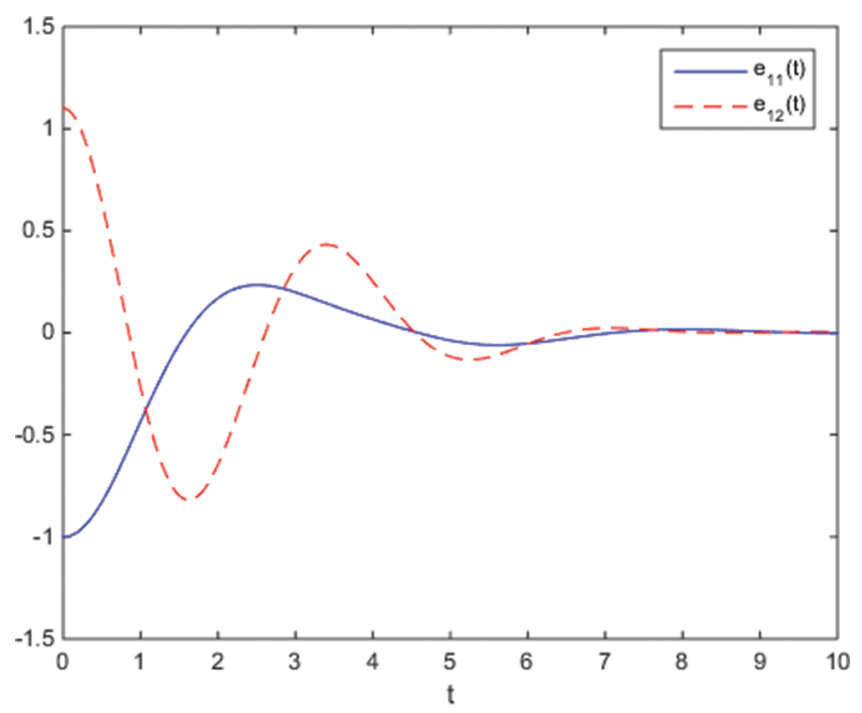

Figure 2 Dynamic behaviors of error signal between drive system (24) and response system (25).
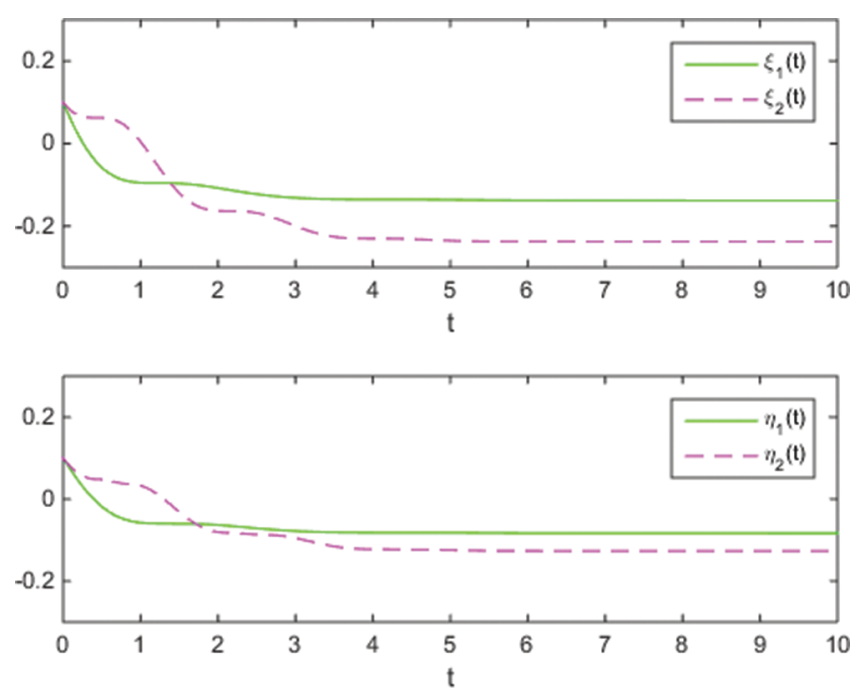

Figure 3 Dynamic behaviors of parameters $\xi_{i}$ and $\eta_{i}(i=1,2)$ in the adaptive controller (6). makes this problem more complicated and challenging. By employing the method of variable transformation, our synchronization criterion is presented in component form, which can be easily verified. It is also worth pointing out that our results comprise some conclusion appeared in the previous literature, as well as reduce restriction on the controller. In the end, a convictive example is proposed to demonstrate the feasibility of the adaptive strategy.

\section{CONFLICT OF INTEREST}

The authors declare that there is no conflict of interests regarding the publication of this article.

\section{AUTHORS' CONTRIBUTIONS}

Cao and Liu conceived and designed the study. Huang wrote the paper. Cao and Liu reviewed and edited the manuscript. All authors read and approved the manuscript.

\section{ACKNOWLEDGMENTS}

This work was partially supported by the National Natural Science Foundation of China under Grants 61573096 and 61833005, the Jiangsu Provincial Key Laboratory of Networked Collective Intelligence under Grant BM2017002. (Corresponding author: Jinde Cao.)

\section{REFERENCES}

[1] M.P. Kenn, L.O. Chua, Neural networks for nonlinear programming, IEEE Trans. Circuits Syst. 35 (1988), 554-562.

[2] Y. Takahashi, Solving optimization problems with variableconstraint by an extended cohen-grossberg model, Theor. Comput. Sci. 158 (1996), 279-341.

[3] R. Parisi, E.D. Di Claudio, G. Lucarelli, G. Orlandi, Car plate recognition by neural networks and image processing, in ISCAS'98 Proceedings of the 1998 IEEE International Symposium on Circuits and Systems (Cat. No. 98CH36187), IEEE, Monterey, CA, USA, 1998, vol. 3, pp. 195-198.

[4] X. Li, J. Cao, Delay-dependent stability of neural networks of neutral type with time delay in the leakage term, Nonlinearity. 23 (2010), 1709.

[5] J. Cao, R. Li, Fixed-time synchronization of delayed memristorbased recurrent neural networks, Sci. Chin. Inf. Sci. 60 (2017), 032201 .

[6] Z. Zhang, Y. Lu, L. Zheng, S. Li, Z. Yu, Y. Li, A new varyingparameter convergent-differential neural-network for solving time-varying convex qp problem constrained by linear-equality, IEEE Trans. Automat. Control. 63 (2018), 4110-4125.

[7] Z. Zhang, L. Zheng, J. Weng, Y. Mao, W. Lu, L. Xiao, A new varying-parameter recurrent neural-network for online solution of time-varying sylvester equation, IEEE Trans. Cybern. 48 (2018), 3135-3148.

[8] S. Rajavel, R. Samidurai, J. Cao, A. Alsaedi, B. Ahmad, Finitetime non-fragile passivity control for neural networks with timevarying delay, Appl. Math. Comput. 297 (2017), 145-158. 
[9] R. Li, J. Cao, Dissipativity analysis of memristive neural networks with time-varying delays and randomly occurring uncertainties, Math. Methods Appl. Sci. 39 (2016), 2896-2915.

[10] X. Li, S. Song, Impulsive control for existence, uniqueness, and global stability of periodic solutions of recurrent neural networks with discrete and continuously distributed delays, IEEE Trans. Neural Netw. Learn. Syst. 24 (2013), 868-877.

[11] I. Stamova, T. Stamov, X. Li, Global exponential stability of a class of impulsive cellular neural networks with supremums, Int. J. Adapt. Control Signal Process. 28 (2014), 1227-1239.

[12] J. Cao, D.W. Ho, X. Huang, Lmi-based criteria for global robust stability of bidirectional associative memory networks with time delay, Nonlinear Anal. Theor. Methods Appl. (2007), 1558-1572.

[13] X. Li, R. Rakkiyappan, N. Sakthivel, Non-fragile synchronization control for markovian jumping complex dynamical networks with probabilistic time-varying coupling delays, Asian J. Control. 17 (2015), 1678-1695.

[14] H. Bao, J.H. Park, J. Cao, Matrix measure strategies for exponential synchronization and anti-synchronization of memristorbased neural networks with time-varying delays, Appl. Math. Comput. 270 (2015), 543-556.

[15] X. Li X. Fu, "Lag synchronization of chaotic delayed neural networks via impulsive control,” IMA J. Math. Control Inf. 29 (2011), 133-145.

[16] W. He, J. Cao, Adaptive synchronization of a class of chaotic neural networks with known or unknown parameters, Phys. Lett. A. 372 (2008), 408-416.

[17] J. Cao, J. Lu, Adaptive synchronization of neural networks with or without time-varying delay, Chaos Interdiscip. J. Nonlinear Sci. 16 (2006), 013133.

[18] J.H. Park, Adaptive control for modified projective synchronization of a four-dimensional chaotic system with uncertain parameters, J. Comput. Appl. Math. 213 (2008), 288-293.

[19] R. Rakkiyappan, R. Sivasamy, X. Li, Synchronization of identical and nonidentical memristor-based chaotic systems via active backstepping control technique, Circuits Syst. Signal Process. 34 (2015), 763-778.

[20] M.A. Cohen, S. Grossberg, Absolute stability of global pattern formation and parallel memory storage by competitive neural networks, IEEE Trans. Syst. Man Cybern. 13 (1983), 815-826.
[21] Q. Zhu, J. Cao, Adaptive synchronization of chaotic cohencrossberg neural networks with mixed time delays, Nonlinear Dyn. 61 (2010), 517-534.

[22] C.-J. Cheng, T.-L. Liao, C.-C. Hwang, Exponential synchronization of a class of chaotic neural networks, Chaos Solitons Fract. 24 (2005), 197-206.

[23] C.-J. Cheng, T.-L. Liao, J.-J. Yan, C.-C. Hwang, Synchronization of neural networks by decentralized feedback control, Phys. Lett. A. 338 (2005), 28-35.

[24] J. Yu, C. Hu, H. Jiang, Z. Teng, Exponential synchronization of cohen-grossberg neural networks via periodically intermittent control, Neurocomputing. 74 (2011), 1776-1782.

[25] Q. Gan, Adaptive synchronization of cohen-grossberg neural networks with unknown parameters and mixed time-varying delays, Commun. Nonlinear Sci. Numer. Simul. 17 (2012), 3040-3049.

[26] B.D. Coleman, G.H. Renninger, Periodic solutions of certain nonlinear integral equations with a time lag, SIAM J. Appl. Math. 31 (1976), 111-120.

[27] D.W. Wheeler, W. Schieve, Stability and chaos in an inertial twoneuron system, Physica D Nonlinear Phenomena. 105 (1997), 267-284.

[28] K. Babcock, R. Westervelt, Stability and dynamics of simple electronic neural networks with added inertia, Physica D Nonlinear Phenomena. 23 (1986), 464-469.

[29] J. Hu, J. Cao, A. Alofi, A.-M. Abdullah, A. Elaiw, Pinning synchronization of coupled inertial delayed neural networks, Cogn. Neurodynamics. 9 (2015), 341-350,

[30] J. Cao, Y. Wan, Matrix measure strategies for stability and synchronization of inertial bam neural network with time delays, Neural Netw. 53 (2014), 165-172.

[31] S. Lakshmanan, M. Prakash, C.P. Lim, R. Rakkiyappan, P. Balasubramaniam, S. Nahavandi, Synchronization of an inertial neural network with time-varying delays and its application to secure communication, IEEE Trans. Neural Netw. Learn. Syst. 29 (2016), 195-207.

[32] M. Prakash, P. Balasubramaniam, S. Lakshmanan, Synchronization of markovian jumping inertial neural networks and its applications in image encryption, Neural Netw. 83 (2016), 86-93. 